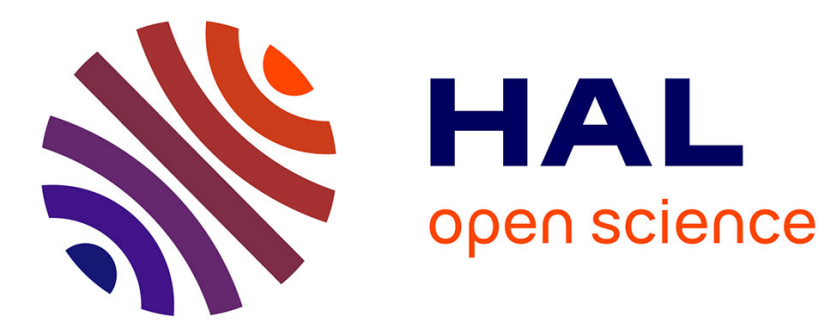

\title{
Level set diagrams of polyhedral objects
}

Francis Lazarus, Anne Verroust

\section{To cite this version:}

Francis Lazarus, Anne Verroust. Level set diagrams of polyhedral objects. SMA'99 Proceedings of the fifth ACM symposium on Solid modeling and applications, ACM, Jun 1999, Ann Arbor, United States. 10.1145/304012.304025 . hal-01224920

\section{HAL Id: hal-01224920 \\ https://hal.inria.fr/hal-01224920}

Submitted on 5 Nov 2015

HAL is a multi-disciplinary open access archive for the deposit and dissemination of scientific research documents, whether they are published or not. The documents may come from teaching and research institutions in France or abroad, or from public or private research centers.
L'archive ouverte pluridisciplinaire HAL, est destinée au dépôt et à la diffusion de documents scientifiques de niveau recherche, publiés ou non, émanant des établissements d'enseignement et de recherche français ou étrangers, des laboratoires publics ou privés. 


\section{Level Set Diagrams of Polyhedral Objects}

\author{
Francis Lazarus* \\ lazarus@sic.univ-poitiers.fr
}

\author{
Anne Verroust ${ }^{\dagger}$ \\ Anne.Verroust@inria.fr
}

\begin{abstract}
Shape descriptors and feature-based representations are of primary interests in the area of solid modeling. They allow us for easier storage, recognition and general treatments of objects. Axial structures such as skeletons are popular shape descriptors which have been widely studied. Most of the studies focus on a particular type of skeleton called the Medial Axis. Medial Axes can be extracted from discrete volumetric data as well as boundary-based representations. In the later case, however, no algorithm is known to perform well and accurately. We propose a new paradigm for constructing one dimensional axial structures associated with a polyhedral object. These structures, called the level set diagrams, are associated with scalar functions defined over the set of vertices of a polyhedron. We study in details the level set diagram associated with the shortest path distance to a source point. This particular association fits nicely into a theoretical framework and presents interesting properties for the purpose of shape description.
\end{abstract}

Keywords: skeleton, cylindrical decomposition, Euler Formula, Dijkstra's algorithm.

\section{Introduction}

We present a new paradigm for constructing one dimensional axial structures, the level set diagrams (LSDs for short), associated with a polyhedral object. These diagrams capture the overall shape as well as the topology of an object. They can be used for deforming or animating an object.

This construction is radically different from the classical Medial Axis [5, 14] which does not necessarily produces a one

* Université de Poitiers. I.R.C.O.M.-S.I.C. SP2MI, Boulevard 3, Teleport 2, B.P. 179, 86960 Futuroscope Cedex, FRANCE

†INRIA Rocquencourt, Domaine de Voluceau, B.P. 105, 78153 Le Chesnay Cedex, FRANCE dimensional structure. Formally, the Medial Axis is the set of the centers of maximal spheres contained in a given object. Algorithms that construct the Medial Axis of a polyhedral surface are generally complex. They start with adding points on the object boundary and computing their Delaunay triangulation [9]. Generally, the resulting Medial Axis needs to be pruned as it may contains numerous small branches [1]. Also, the Medial Axis can be composed of pieces of curves as well as pieces of surfaces. By construction, however, LSDs are simple one dimensional graphs. They relate to the level sets of scalar functions defined over the vertices of a polyhedral surface. More precisely, the points of an LSD correspond to "average points" associated with the connected components of the level sets of a given scalar function. Figure 1 shows an LSD of a threefingered shape.

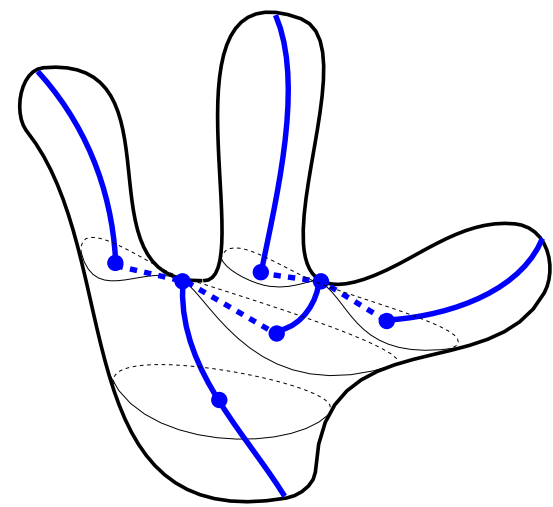

Figure 1: An LSD (bold lines) of a an object is defined by the centers of the connected components of the level sets (thin lines) of a given function. The dashed bold lines indicate joints between LSD branches.

Shinagawa et al. $[19,20]$ have introduced a similar diagram in the context of medical and geographical applications. They call their diagram the Reeb graph in reference to a classical topological graph associated with level sets of a Morse function ${ }^{1}$, $f$, defined on a smooth surface $[15,10]$. The vertices of the Reeb graph correspond to critical points of the function $f$, that is points where the gradient of $f$ is null.

Shinagawa et al. are mainly interested in encoding the topological structure of a terrain while the LSD is a geometrical skeleton

\footnotetext{
${ }^{1}$ A Morse function, on a surface, is a twice differentiable function whose Hessian - the Jacobian of the gradient in a suitable coordinate system - has a non-zero determinant everywhere its gradient vanishes.
} 
that describes both the topology and the geometry of an object.

LSDs depend on the particular function chosen to compute the level sets. Shinagawa et al. use the height function (the zcoordinate), relatively to a given orientation of the object to define their diagram. This is particularly meaningful for terrain data as critical points correspond to peaks, pits or passes of the terrain. We show that in our case the shortest path distance to a source point is often a better choice of function especially for describing the geometry of tubular and branching objects.

The two major components of our approach are the study of the level sets of a function defined on a polyhedron and the use of the shortest path distance to a source point to define such a function. de Berg et van Kreveld [7] studied the structure of the level sets of the height function on a polyhedral terrain. As in $[19,20]$, the structure of the level sets is recorded into a tree analogous to the Reeb graph. In [7], it is shown that this tree, called the contour tree, can be constructed in $\mathcal{O}(n \log n)$ time. In [22], a simpler construction of the contour tree is provided for general functions (not only height functions) defined on general polyhedra (not only terrains).

An LSD is a geometric embedding of the contour tree where each point of the contour tree is mapped to the isobarycenter of the corresponding level set contour.

In [12], we used the shortest path distance to a source point to define a function on the vertices of a polyhedron. The same function is used by Axen and Edelsbrunner [2] in a very different context. In this work, the topology of surfaces is explored by a wave traversal to produce sounds in relation with the topology of the surface. The waves precisely correspond to level sets of the shortest path distance to a source point. Note, however, that Axen and Edelsbrunner consider topological distances - edges have unit length - rather than Euclidean distances.

Section 2 presents some basic definitions and properties of the level sets of a function defined on a triangulated polyhedron. In Section 3, we describe the evolution of a the level-sets as the level is varied. This description is based upon the notion of index of a vertex. Section 4 studies the level sets of the shortest path distance to a source point. This study is used in Section 5 to define efficient algorithms that construct the LSD. Results are commented in Section 6. Finally, we discuss future works and conclude in Section 7.

\section{Level Sets of Functions}

This section provides some definitions and notation that are important to the construction and properties of the LSD. In the following, we only consider LSDs of triangulated polyhedra.

For a given triangulated polyhedron, $\mathcal{P}$, we consider a function, $f$, defined over its vertices $v_{1}, \ldots, v_{n}$ so that two adjacent vertices are given two different values. Actually, as noticed in [20], all that is required is to tell which of any two adjacent vertices gets the smaller value. In practice, we can order arbitrarily the vertices having the same value so that the above requirement can be alleviated.
Definition 1 An edge $\left(v_{i}, v_{j}\right)$ is said to be a cross-edge relatively to a level $l$ if

$$
f\left(v_{i}\right)<l<f\left(v_{j}\right)
$$

Similarly, a face $\left(v_{i}, v_{j}, v_{k}\right)$ is said to be a cross-face relatively to a level $l$ if the three values of its vertices encompass $l$, that is

$$
\begin{gathered}
f\left(v_{i}\right)<l<f\left(v_{j}\right)<f\left(v_{k}\right), \text { or } \\
f\left(v_{i}\right)<f\left(v_{j}\right)<l<f\left(v_{k}\right) .
\end{gathered}
$$

Remark that a cross-face has exactly two cross-edges.

Definition 2 The dual graph of $\mathcal{P}$ is the graph whose vertices are the faces of $\mathcal{P}$ and whose edges correspond to pairs of incident faces.

The preceding remark shows that a cross-face (relatively to a level $l$ ) is adjacent to exactly two cross-faces in the dual graph (see Figure 2).

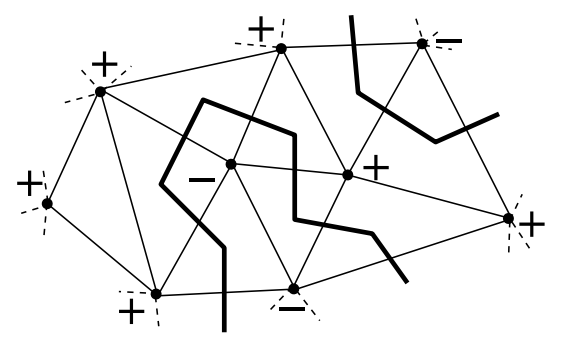

Figure 2: A triangulation with its cross-adjacency graph (bold lines) for a given level $l$. Vertices with values greater (smaller) than $l$ are marked with a "+" (a "-").

Property 1 For any level $l$, distinct from the values $f\left(v_{1}\right), \ldots, f\left(v_{n}\right)$, the set of cross-faces constitutes a set of disjoint cycles in the dual graph.

This property is to be compared with the fact that a level set of a Morse function defined on a closed smooth surface is composed of disjoint closed curves, if that level set contains no critical point.

These cycles of cross-faces define closed polygonal contours on the polyhedron $\mathcal{P}$. For this, we associate with each cross-edge $\left(v_{i}, v_{j}\right)$ the point

$$
p_{i j}(l)=\frac{l-f\left(v_{i}\right)}{f\left(v_{j}\right)-f\left(v_{i}\right)} v_{i}+\frac{f\left(v_{j}\right)-l}{f\left(v_{j}\right)-f\left(v_{i}\right)} v_{j} .
$$

If $\left(v_{i}, v_{j}, v_{k}\right)$ is a cross-face, we can draw the line $p_{i j}(l) p_{i k}(l)$ as on Figure 3.

Definition 3 The geometric realization of a level set is the set of polygonal contours obtained by drawing the lines $\left(p_{i j}(l) p_{i k}(l)\right)$ in every cross-faces. We will note $\mathcal{C}(l)$ the realization of the level set of level $l$. 


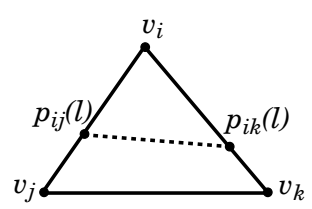

Figure 3: Segment $\left(p_{i j}(l) p_{i k}(l)\right)$ is the geometric realization of the cross-face $\left(v_{i}, v_{j}, v_{k}\right)$.

These polygonal contours are not necessarily planar. They will be further "averaged" in order to construct the LSD.

If $l_{1}<l_{2}$ are two levels, such that no vertex $v$ verifies $l_{1} \leq$ $f(v) \leq l_{2}$, then the two corresponding level sets are traversed by the same cross-faces. It follows that $\mathcal{C}\left(l_{1}\right)$ can be deformed continuously into $\mathcal{C}\left(l_{2}\right)$ and vice versa. In other words, $\mathcal{C}\left(l_{1}\right)$ and $\mathcal{C}\left(l_{2}\right)$ are homotopic. In particular, they have the same number of contours.

This result will be strengthen in the next section. As we will see, the contours are still homotopic when the level $l$ passes through a value $f(v)$, as long as $v$ is a regular vertex.

\section{Vertex Index and Euler Formula}

The LSD encodes the set of contours $\mathcal{C}(l)$ as $l$ evolves. Branching of the LSD can only occur when $\mathcal{C}(l)$ changes of homotopy type. It is thus important to know for which value of $l$ the topology of the polygonal contours $\mathcal{C}(l)$ is modified. As we just saw, this can only happen when $l$ equals $f(v)$ for some vertex $v$ of the polyhedron $\mathcal{P}$.

In order to describe the topological change at a vertex $v$ we introduce the index of a vertex $v$. Let $w_{1}, w_{2}, \ldots, w_{k}$ be the $k$ neighbors of $v$ enumerated counterclockwise around $v$. As Takahashi et al. [20], we consider the number of sign changes, $S g c_{f}(v)$, in the sequence $\left(f\left(w_{1}\right)-f(v), f\left(w_{2}\right)-\right.$ $\left.f(v), \ldots, f\left(w_{k}\right)-f(v), f\left(w_{1}\right)-f(v)\right)$. The index of $v$ is defined as

$$
\operatorname{Ind}_{f}(v)=1-\frac{S g c_{f}(v)}{2}
$$

Figure 4 shows a vertex with index -1 . This index tells how $f$

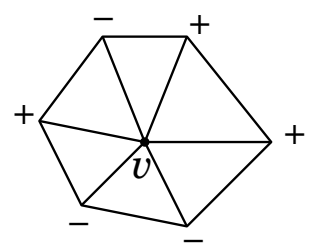

Figure 4: A “+” (a "-”) indicates a $v$ neighbor, $w$, such that $f(w)$ is greater (smaller) than $f(v)$. The number of sign changes $S g c_{f}(v)$ counts the number of "+" and "-" sequences. is changing around $v$. It actually records part of the topology of $\mathcal{P}$ itself. By summing the indices over all the vertices, we get the very nice formula

\section{Property 2}

$$
\sum_{v \in \mathcal{P}} \operatorname{Ind}_{f}(v)=\chi(\mathcal{P})
$$

where $\chi(\mathcal{P})=n_{V}-n_{E}+n_{F}$ is the Euler characteristic of $\mathcal{P}$ and $n_{V}, n_{E}$ and $n_{F}$ are respectively the number of vertices, edges and faces of $\mathcal{P}$.

proof: $S g c_{f}(v)$ counts the number of triangles $\left(v_{i}, v, v_{j}\right)$ incident to $v$ such that $f(v)$ is between $f\left(v_{i}\right)$ and $f\left(v_{j}\right)$. Since every triangle has exactly one vertex for which this occurs the sum of the $S g c_{f}(v)$ for all the vertices counts the total number of triangles:

$$
\sum_{v \in \mathcal{P}} S g c_{f}(v)=n_{F}
$$

so that

$$
\sum_{v \in \mathcal{P}} \operatorname{Ind}_{f}(v)=n_{V}-\frac{1}{2} n_{F} .
$$

As $\mathcal{P}$ is a triangulation, we have $3 n_{F}=2 n_{E}$ or equivalently $n_{F}=2\left(n_{E}-n_{F}\right)$. It follows that

$$
\sum_{v \in \mathcal{P}} \operatorname{Ind}_{f}(v)=n_{V}-n_{E}+n_{F}=\chi(\mathcal{P})
$$

The same formula can be found in [3] and [11] in a slightly different context. Takahashi et al. [20] implicitly refer to a similar formula but they only consider vertices for which $S g c_{f}(v)$ equals 0 or 4 . In fact, Takahashi et al. directly refer to Morse theory. In this theory a critical point may only get three different index values, two of which corresponding to local extrema. Another effective analogy is provided with the notion of index of a critical point of a gradient vector field. A critical point of a vector field is a (isolated) point where the vector field is null. Very roughly speaking the index of a critical point is the number of revolutions made by the vector field while turning around that point [11]. In order to show the analogy with our notion of index, we first orient the edges according to the $f$ values of their endpoints. This orientation corresponds to the gradient of $f$ along the edges. Note that a triangle cannot have its edges oriented clockwise nor counterclockwise as shown in Figure 5. Then, in each triangle of $\mathcal{P}$ we draw a continuous vector field, as sketched on Figure 5, so that the direction of the vector field on the triangle boundary agrees with the orientation of its edges. Now, the vertices of $\mathcal{P}$ are the critical points of this vector field and it can be shown that their index, relatively to the vector field, coincides with our notion of index. The above Euler formula thus corresponds to a classical summation formula over the index of the critical points of a continuous vector field [11].

The index of a vertex $v$ describes how the level set realization $\mathcal{C}(l)$ is modified around $v$, as the level $l$ is raised from $f(v)-\epsilon$ to $f(v)+\epsilon$, for a sufficiently small $\epsilon$. 

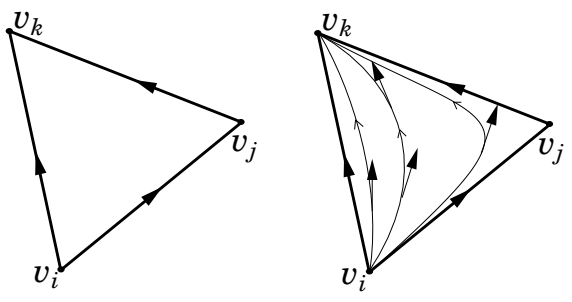

Figure 5: On the left: Orientation of the edges of a triangle induced by $f\left(f\left(v_{i}\right)<f\left(v_{j}\right)<f\left(v_{k}\right)\right)$. On the right: The tangent vectors to the oriented curves defines a vector field compatible with the edge orientation.

If $\operatorname{Ind}_{f}(v)$ is null, then the $v$ neighbors are partitioned into two connected sets $\left\{v_{-} \mid f\left(v_{-}\right)<f(v)\right\}$ and $\left\{v_{+} \mid f\left(v_{+}\right)>\right.$ $f(v)\}$ as shown in Figure 6 on the left. $\mathcal{C}(f(v)+\epsilon)$ is deduced
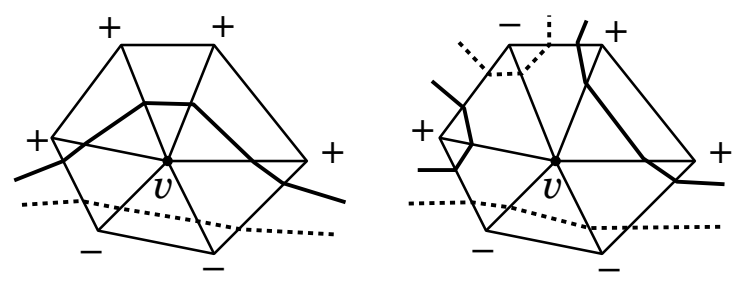

Figure 6: The neighborhood of a vertex $v$ in two different configurations. a '+' ('-') indicates a $v$ neighbor, $w$, such that $f(w)$ is greater (smaller) than $f(v)$. The level set realization is shown for a level slightly below $f(v)$ (dotted curve) and slightly above $f(v)$ (plain curve). The index of $v$ is 0 on the left and -1 on the right.

from $\mathcal{C}(f(v)-\epsilon)$ by substituting points on cross-edges of the type $\left(v, v_{-}\right)$to points on cross-edges of the type $\left(v, v_{+}\right)$. The change reduces to a sweep and a chain substitution of crossedges so that $\mathcal{C}(f(v)+\epsilon)$ is still homotopic to $\mathcal{C}(f(v)-\epsilon)$.

Several chain substitutions may occur at the same time when different non adjacent vertices get the same value $f(v)$.

Definition 4 A vertex with a null index is said to be regular and critical otherwise. Similarly the value $f(v)$ of a critical vertex is called a critical value. Any other value is said to be regular.

Note that a positive index $(+1)$ indicates a local extremum. A critical vertex which is not an extremum is called a saddle vertex. When traversing a critical value the topology of the level set is changing. The evolution of the level set depends on the index of the corresponding critical vertex (see Figure 6). The precise topological change not only depends on the index but also on the whole configuration of the level set. Figure 7 shows two possible changes in the level sets that depend on the configuration of $\mathcal{C}(f(v)-\epsilon)$. As will be shown in the next section the topological changes of the level sets can be deduced directly

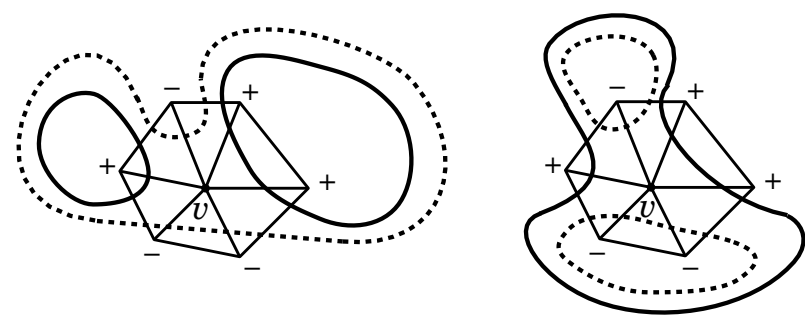

Figure 7: Two configurations of a vertex with index -1 . The number of connected components of the level set increases on the left while it decreases on the right as the level is augmented from below $f(v)$ (dotted curve) to above $f(v)$ (plain curve).

from the vertex indices when considering the shortest path distance to a source point on a genus- 0 polyhedron.

\section{The Shortest Path Distance to a Source Point}

Up to this point we have not considered any specific $f$. As we want the LSD to represent the object geometry, the definition of $f$ should involve the geometry of $\mathcal{P}$. Takahashi et al. use the height function [20], so that any vertex is associated its own zcoordinate in a suitable coordinate system. In this case, the level sets correspond to cross-sections between $\mathcal{P}$ and $x y$-planes. As noticed in the introduction this choice is relevant for terrain data as the critical vertices correspond to peaks, passes or pits of the terrain. However, for other classes of objects it may happen that the height function does not give an intuitive LSD in any coordinate system. Figure 13 shows a self-intersecting tubular object whose "natural" axis could not be determined with any height function. A better choice for the level sets is provided with the moving front of some flow emanating from a tip of the tube. Such fronts correspond to the level sets of the geodesic distance to a tip of the tube. The computation of the geodesic distance to a source point on a polyhedron has been extensively studied (see [16]). We prefer to compute the shortest path distance relatively to the graph induced by the edges of a polyhedron. It provides an approximation, sufficient for our purpose, of the geodesic distance. It further allows us to simplify and accelerate the computation and fits nicely into a theoretical framework.

In the following, we study the level sets of the shortest path distance to a given source point. We will note the function $d_{s}$ instead of $f$. For any vertex $v, d_{s}(v)$ is the length of a shortest path to a given source point $s$. (The choice of $s$ will be discussed in the results section.) $d_{s}$ can be computed efficiently with a modified breadth-first search starting from the source and using the classical Dijkstra's algorithm [6]. Dijkstra's algorithm makes use of a priority queue to select the next vertex to be processed in the breadth-first search. Dijkstra's algorithm also returns for any vertex $v$ a pointer $\pi(v)$ to the preceding vertex on a shortest path linking $v$ to the source $s$. By iterating the $\pi$ pointer we can follow a strictly decreasing path from any vertex to the source. 
$d_{s}$ presents interesting properties that allows for easy computation of the associated LSD. First of all, the source point $s$ is the only local minimum of $d_{s}$. This means that from any vertex we can draw a path of edges leading to the source point with strictly decreasing vertex $d_{s}$-values. To obtain further nice properties we will assume from now on that the polyhedron $\mathcal{P}$ has a 0 -genus, that is $\mathcal{P}$ is topologically equivalent to a triangulated sphere.

With this assumption, we get the following property that will provide a complete description of the topological change when the level passes through a critical value.

Property 3 The set of cross-faces incident to $v$ for a level $l$ lower than $d_{s}(v)$ belong to a unique cycle.

proof: Since $\mathcal{P}$ has a 0 -genus, the realization of any level set cycle splits $\mathcal{P}$ into two connected components. If such a cycle contains a cross-face incident to $v$, then one of these two components must contains $v$ while the other contains the source point. This is easily seen from the above decreasing path property (see also Figure 8). Now, any decreasing path $p$ from $v$ to

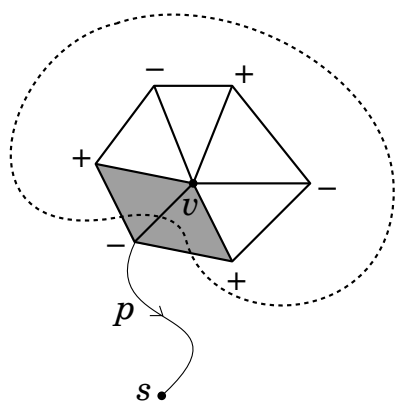

Figure 8: The path $p: v \rightarrow s$ meets the realization of any level set cycle containing one or more cross-faces (shaded on the Figure) incident to $v$ once and only once.

$s$ should meet every cycle containing a cross-face incident to $v$. Since $p$ meets the whole level set $\mathcal{C}(l)$ exactly once, there must be only one such cycle $\square$

From this property it is seen that the configuration of Figure 7 on the right cannot occur. Also, if $n_{c}(l)$ counts the number of cycles of $\mathcal{C}(l)$, then for any vertex $v$, we have

$$
n_{c}\left(d_{s}(v)+\epsilon\right)=n_{c}\left(d_{s}(v)-\epsilon\right)-\operatorname{Ind}_{d_{s}}(v),
$$

since any chain of $v$ neighbors further than $v$ from the source gives rise to a new cycle (see Figure 7 left).

Property 3 prevents the LSD from having loops. The vertex with the highest distance $d_{s}$ on a loop would indeed have two branches going down the source which would contradict property 3. Since the LSD is connected (just follow a decreasing path to the source from any vertex on a cycle), the LSD must be a tree.

The tree structure of the LSD is recorded into a contour tree [22] $\mathcal{T}_{s}(\mathcal{P})$ whose root, internal nodes and leaves are respectively the source $s$, the saddle vertices, and the local maxima.
The lines of $\mathcal{T}_{s}(\mathcal{P})$ link critical vertices and correspond to cylindrical parts of $\mathcal{P}$. Figure 9 shows a simple example.
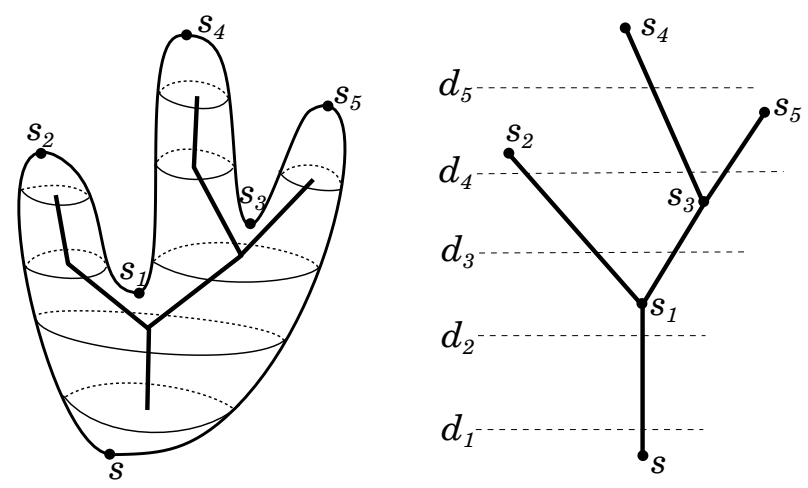

Figure 9: The LSD is an embedding of the tree $\mathcal{T}_{s}(\mathcal{P})$. On the left the LSD is computed from five level sets of $d_{s}$ corresponding to the distances $d_{1}, \ldots, d_{5}$.

In practice, we will use its tree structure to construct the LSD.

\section{Algorithms}

We present algorithms for computing the tree $\mathcal{T}_{s}(\mathcal{P})$ and the LSD associated with the distance $d_{s}$. To clarify the presentation we assume that no two vertices of $\mathcal{P}$ are at the same distance from the source. In case this would happen, we can still arbitrarily order the vertices having the same distance so that we can compare any pair of adjacent vertices. In [2], this kind of degeneracies is treated by first performing a barycentric subdivision. However, this technique does not apply here as we are not considering topological distances. Also, the barycentric subdivision does not solve all the degeneracies (i.e. adjacent critical vertices with the same distance) and further arbitrary choices must be taken.

We also assume that the neighbors of a vertex, the incident faces to an edge, and the edges and vertices of a face can be accessed in constant time $\mathcal{O}(1)$. The winged-edge data structure $[4,25]$, for instance, fulfills all these requirements.

To construct the LSD, we need to sweep the level from zero to its maximum value $\max d_{s}$ and compute the "average" point of various level set cycles.

The set of cross-faces contained in a level set changes whenever the level passes trough a vertex value. The number of different level sets is thus equal to the number of vertices. Since most of the vertices have to be regular most of these level sets will have the same topology. Also, polyhedral objects may have hundreds or thousands of vertices, and we do not need that many level sets to construct the LSD. In practice, we fix the number of levels (from 16 to 50 in our examples) and select the levels uniformly in the range $\left[0, \max d_{s}\right]$. For each selected levels, we must compute the corresponding level set realization. This 
requires finding a starting cross-edge in each cycle of the level set. Then, from faces incident to these starting cross-edges, we can walk the dual graph of $\mathcal{P}$ along the level set and determine all the level set cycles.

These starting cross-edges play the role of what van Kreveld et al. [22] call a seed set. In our case there is no need to compute such a seed set. The tree structure $\mathcal{T}_{s}(\mathcal{P})$ of the LSD actually provides an efficient tool to determine a starting edge in each cycle of any level set. Precisely, we build the LSD while traversing the lines of $\mathcal{T}_{s}(\mathcal{P})$. For each line $\left(s_{i}, s_{j}\right)$ of $\mathcal{T}_{s}(\mathcal{P})$ we can obtain a starting cross-edge in any cycle contained in the associated cylindrical part. This is done with a decreasing path starting at $s_{j}$ as shown in Figure 10.

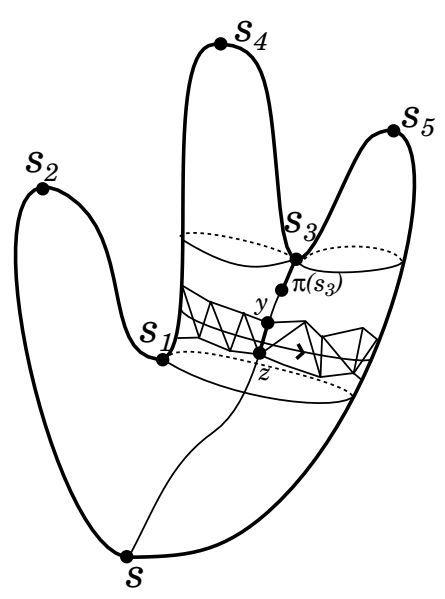

Figure 10: The $\pi$ pointer provided by the Dijkstra's algorithm is used to find a starting cross-edge $(y, z)$ of a cycle contained in the cylindrical region between $s_{1}$ and $s_{3}$.

As the construction algorithm is guided by the tree structure $\mathcal{T}_{s}(\mathcal{P})$, we can assure that we do not miss any cycle in a level set and that each cycle is computed only once.

The overall LSD construction algorithm is thus based on two steps: in a first step, we build the tree $\mathcal{T}_{s}(\mathcal{P})$, and in a second step, we compute the LSD itself.

\subsection{Computing $\mathcal{T}_{s}(\mathcal{P})$}

The construction of $\mathcal{T}_{s}(\mathcal{P})$ is based on Dijkstra's algorithm. It is performed while traversing the adjacency graph of $\mathcal{P}$. During this traversal, we compute the shortest path distance $d_{s}$ of the visited vertices as well as the $\pi$ pointer to the preceding neighbor on a shortest path. As for the classical Dijkstra's algorithm, we maintain a priority queue with respect to the distance. This priority queue contains the vertices adjacent to the already visited vertices, but whose distance have not yet been fixed by the algorithm. Since in the Dijkstra's algorithm vertices are processed in their order of distance, this traversal amounts to sweep a level set from distance zero to the maximum distance.
The construction of $\mathcal{T}_{s}(\mathcal{P})$ is directly inspired from the contour tree algorithm in [22]. Starting from the root, we visit the vertices of $\mathcal{P}$ until we encounter a singular vertex $v$. At this point, we create a line $(s, v)$ of $\mathcal{T}_{s}(\mathcal{P})$. If $v$ is a local maximum the construction is stopped and $\mathcal{T}_{s}(\mathcal{P})$ reduces to the single line $(s, v)$. Otherwise, $v$ must be a saddle vertex, and we split the priority queue into $1-\operatorname{Ind}_{d_{s}}(v)$ subqueues corresponding to the branches of $\mathcal{T}_{s}(\mathcal{P})$. In order to represent several branches (i.e. subqueues) in the priority queue, we label every vertex $v$ in the queue with a contour number $c(v)$. Splitting a contour in the queue amounts to replace the contour number of its vertices by traversing each new contour. We also maintain a contour table $C T$ containing for every contour $i$ the saddle vertex $C T[i]$ that gave birth to this contour branch during the sweep.

The algorithm proceeds as follows

0 (Initialize)

$Q:$ a priority queue of vertices w.r.t. $d_{s}$

for each vertex $v$ of $\mathcal{P}$

Mark $v$ as unprocessed.

$d_{s}(s)=0$

$\pi(s)=N U L L$

$Q=\{s\}$

Insert $s$ as the root of $\mathcal{T}_{s}(\mathcal{P})$

$C T[1]=s$

$n_{c}=1\left(n_{c}\right.$ is the number of contours in the queue)

$c(v)=1$

\section{begin}

1 (Main loop)

1 while $Q$ is not empty do

begin

Extract $v=$ head of $Q$ and dequeue $Q$

Mark $v$ as processed

$5 \quad$ for $v_{i}$ adjacent to $v$ and marked unprocessed do

if $v_{i}$ is not in $Q$ then

begin

Set $d_{s}\left(v_{i}\right)$ to $d_{s}(v)+\left\|v-v_{i}\right\|$

Set $\pi\left(v_{i}\right)$ to $v$

10

Set $c\left(v_{i}\right)$ to $c(v)$

Insert $v_{i}$ into $Q$

end

else if $d_{s}\left(v_{i}\right)>d_{s}(v)+\left\|v-v_{i}\right\|$ then

begin

15

Change $d_{s}\left(v_{i}\right)$ to $d_{s}(v)+\left\|v-v_{i}\right\|$

Change $\pi\left(v_{i}\right)$ to $v$

Change $c\left(v_{i}\right)$ to $c(v)$ end

Compute the index $\operatorname{Ind}(v)$

20

if $\operatorname{Ind}(v)=1$ ( $v$ is a local maximum) then

Insert line $(C T[c(v)], v)$ into $\mathcal{T}_{s}(\mathcal{P})$ with $v$ as a leaf

else if $\operatorname{Ind}(v)<0$ ( $v$ is a saddle point) then

begin

Insert line $(C T[c(v)], v)$ into $\mathcal{T}_{s}(\mathcal{P})$

$25 \quad$ Split contour $c(v)$ into $1-\operatorname{Ind}(v)$ subcontours

corresponding to the cycles incident to $v$

of the level set $\mathcal{C}\left(d_{s}(v)+\epsilon\right)$

Use the old contour number $c(v)$ and the new numbers

30

$n_{c}+1, \ldots n_{c}-\operatorname{Ind}(v)$ for the new contours

$C T[c(v)]=v$

for $1 \leq j \leq-\operatorname{Ind}(v)$ do

$C T\left[n_{c}+j\right]=v$

$n_{c}=n_{c}-\operatorname{Ind}(v)$ 
From statement 2 it is seen that each iteration of the main loop corresponds to the sweep of all the level set cycles with respect to the distance $d_{s}$ and it maintains the labelling of the vertices according to the level set cycles already encountered.

Analysis In the following we sketch some proof for correctness of the algorithm and analyze its complexity.

Statement 1 Ind $(v)$ can be correctly computed at line 18 of the main loop.

In the classical Dijkstra's algorithm vertices are dequeued in distance order [6]. This is still true in our case as we use Dijkstra's algorithm without changing the order of processing of the vertices.

When dequeuing a vertex $v$ (line 3), all the adjacent vertices with a distance smaller than $d_{s}(v)$ have already been processed while all the other adjacent vertices must lie in the queue with their distance set to a value greater than $d_{s}(v)$. It follows that $\operatorname{Ind}(v)$ can be correctly computed.

Statement 2 When $Q$ is dequeued (line 3 of the main loop), it contains the extremity of all the cross-edges, relatively to the level $d_{s}(v)-\epsilon$, and these extremities are labelled according to the connected components, or contours, of the level set $d_{s}(v)-$ $\epsilon$.

When $Q$ is dequeued in line 3, the origin of every cross-edge, relatively to the level $d_{s}(v)-\epsilon$, obviously lies at a distance smaller than $d_{s}(v)$ from the source. According to the order of processing, all these origins must have been processed earlier. It follows that the extremity of these cross-edges have been enqueued at line 11 ( $\epsilon$ is chosen so that no vertex other than $v$ has its distance in the interval $\left[d_{s}(v)-\epsilon, d_{s}(v)\right]$ ).

Until the first saddle vertex is processed, all the vertices in $Q$ have the same label, 1 , and we know that the current level set is composed of a single contour.

When a local maximum is encountered, the corresponding contour simply disappears from the queue. When a saddle vertex $w$ is encountered, the corresponding contour $c(w)$ can only split into $1-\operatorname{Ind}(w)$ contours, according to Section 4. Lines 24-31 of the main loop precisely performs this split.

To do so, we walk along each cycle of $\mathcal{C}\left(d_{s}(w)+\epsilon\right)$ corresponding to a chain of +'s (see Figure 7 Right) and change the label $c()$ of the extremity of the traversed cross-edges.

By construction, $Q$ contains:

- either the vertices which had a label different from $c(w)$ just before $w$ is dequeued,

- or the upper extremities of all the cross-edges relatively to the $1-\operatorname{Ind}(w)$ contours of level $d_{s}(w)+\epsilon$ and these extremities have been labelled according to their corresponding contours.

Then $Q$ is further dequeued and the statement follows by induction on the number of singular vertices.

Statement 3 The main loop correctly computes the tree $\mathcal{T}_{s}(\mathcal{P})$.
When a singular vertex $w$ is dequeued, the branch containing the contour $c(w)$ (more precisely, the contour just below $c(w)$ ) either splits or terminates. At this point, we must create a line in $\mathcal{T}_{s}(\mathcal{P})$. This line must have $w$ as its extremity and the saddle vertex that gave birth to this branch as its origin. By construction this origin is precisely $C T[c(w)]$ and the branch is correctly taken into account at line 21 or 24 of the main loop.

From Dijkstra's algorithm all the vertices of $\mathcal{P}$ must be processed so that the main loop visits the entire tree.

The complexity of the algorithm reduces to the complexity of the Dijkstra's algorithm plus the time for splitting the queue. Using a Fibonacci heap for the priority queue, Dijkstra's algorithm requires time $\mathcal{O}(n \log n)$ for a polyhedron with $n$ vertices $^{2}$. A contour $c(v)$ must be split each time a saddle vertex is met. The total time spent for splitting the queue is thus bounded by $n_{s} \lambda_{\max }$, where $n_{s}$ counts the number of saddle vertices and $\lambda_{\max }$ is the largest number of cross-edges in a contour. Hence, the computation of $\mathcal{T}_{s}(\mathcal{P})$ requires time $\mathcal{O}\left(n \log n+n_{s} \lambda_{\text {max }}\right)$. $n_{s}$ and $\lambda_{\max }$ can be as large as $\mathcal{O}(n)$ so that this analysis may lead to an $\mathcal{O}\left(n^{2}\right)$ complexity in the worst case. When all splits are simple (i.e. the contour $c(v)$ can only split into two parts $c_{1}$ and $c_{2}$ ), van Kreveld et al. [22] propose to reduce the cost for splitting by traversing the smaller of $c_{1}$ and $c_{2}$ rather than traversing both of them. This is done by tandem search, that is by traversing the two contours $c_{1}$ and $c_{2}$ in parallel until one contour, say $c_{1}$, is entirely traversed. Then, $c_{1}$ is traversed again and the label of its vertices are changed accordingly. This way, the cost for splitting $c(v)$ is proportional to the size of $c_{1}$ (the smaller of $c_{1}$ and $\left.c_{2}\right)$. This leads to an $\mathcal{O}(n \log n)$ time algorithm to compute the contour tree $\mathcal{T}_{s}(\mathcal{P})$ (see [22] for a proof). The same complexity can be obtained for non simple splits by extracting all but the largest contour from the contour $c(v)$ at a branching node.

\subsection{Computing the LSD}

The LSD constitutes a geometric embedding of $\mathcal{T}_{s}(\mathcal{P})$. In practice, we discretize the level interval $\left[0, \max d_{s}\right]$ into a set $\left\{l_{1}, l_{2}, \ldots, l_{r}\right\}$ of $r$ levels and compute the average point of each cycle of the level set realizations $\mathcal{C}\left(l_{1}\right), \ldots, \mathcal{C}\left(l_{r}\right)$. The algorithm proceeds by traversing the tree $\mathcal{T}_{s}(\mathcal{P})$. For each line of the tree we compute and average the cycles of level $l_{1}, \ldots, l_{r}$ contained in the corresponding cylindrical part.

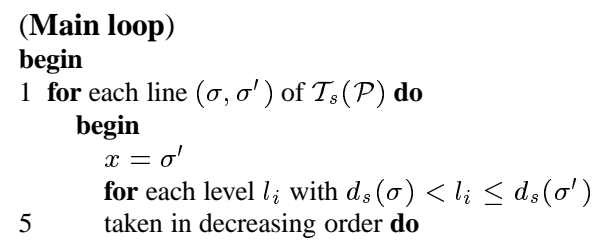

${ }^{2}$ Dijkstra's algorithm works with any connected graph in time $\mathcal{O}(n \log n+p)$ if $p$ is the number of edges. 


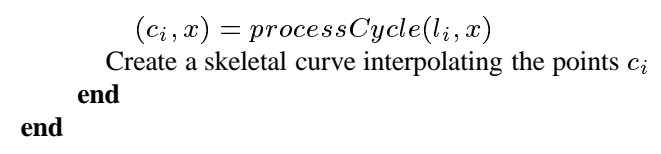

procedure processCycle $(l, z)$

begin

$1 \quad y=z$

$z=\pi(y)$

while $d_{s}(z)>l$ do

begin

$5 \quad y=z$

$z=\pi(y)$ end

Traverse the cycle of level $l$ from cross-edge $(y, z)$ and obtain a polygonal contour realization $C$

10 Compute $c$ as the barycenter of $C$ end

return $(c, y)$

Figure 10 summarizes the construction of a cycle in processCycle. In line 10 of process Cycle we compute the barycenter of the polygonal curve $C$ rather than the barycenter of its vertices. If $C=\left(p_{1}, p_{2}, \ldots, p_{k}\right)$ then

$$
c=\sum_{j=1}^{k}\left\|p_{j} p_{j+1}\right\| \frac{p_{j}+p_{j+1}}{2} / \sum_{j=1}^{k}\left\|p_{j} p_{j+1}\right\|
$$

This formula is independent of the discretization level and avoids the artifacts caused by non-uniform meshes.

Remark that in line 4 of the main loop we replaced the inequalities $d_{s}(\sigma)<l_{i}<d_{s}\left(\sigma^{\prime}\right)$ involved in the definition of a crossedge by the inequalities $d_{s}(\sigma)<l_{i} \leq d_{s}\left(\sigma^{\prime}\right)$. This avoids precision problems when $l_{i}$ equals $d_{s}(v)$ for some vertex $v$. In such a case, the topology of the level set will be the same as the topology of $\mathcal{C}\left(l_{i}-\epsilon\right)$, though the embedding might have a singular point at vertex $v$.

In line 7 of the main loop we can use a simple polygonal curve or a curve fitting algorithm $[17,8]$. Note that our algorithm provides a curve for each branch of the LSD but does not tell how the branches are geometrically linked together. The problem is that the barycenter becomes discontinuous each time a cycle is split into several cycles. In other words, the position of the barycenter of every new cycle changes abruptly from the position of its "parent" cycle. This branching information is yet important for animation and deformation purposes. Therefore, we keep virtual links between the branches according to the tree structure. In the Figures of the results Section branching links are visualized with line segments.

\section{Results}

We present and discuss the results of applying our LSD algorithm to a number of polyhedral objects.

Source point location. In our current implementation the source point can be selected either automatically or interactively. In the former case we apply a heuristic based on a simple

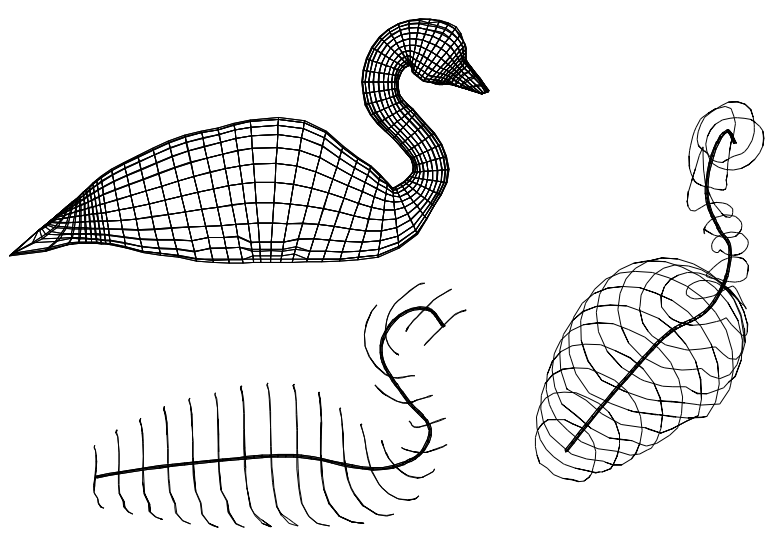

Figure 11: A swan and its LSD. The swan is composed of 1728 faces. 20 level lines have been built from $5 \%$ to $90 \%$ of the maximal distance from the source point which is located at the extremity of its beak (indicated by a white triangle on figure 12). The LSD was obtained in less than a second on a SGI INDIGO2.

algorithm to find a diameter of a tree. A diameter of a tree is a pair of vertices separated by the largest possible distance in the tree. Intuitively, the two vertices of a diameter are located at the tip of a longest branch of the tree. The following procedure determines a tree diameter:

1. Pick a vertex $x$ at random in the tree,

2. Find $y$ such that $d_{x}(y)$ is maximal,

3. Find $z$ such that $d_{y}(z)$ is maximal.

It is relatively easy to show that $(y, z)$ is a diameter. In our case we keep the two first steps of the procedure to find a source point $s(=y)$ which corresponds to the tip of some long branch of our polyhedral object. The source point was selected automatically on Figures 11, 15, and 16.

The shortest path distance is continuous with respect to the source point since

$$
\left|d_{s}(x)-d_{s^{\prime}}(x)\right| \leq d_{s}\left(s^{\prime}\right)=d\left(s, s^{\prime}\right) .
$$

As a consequence, the LSD is stable with respect to the source point location, as illustrated in Figure 12. Note that the 0-level set coincides with the source point. In order to avoid small deviations due to the precise location of the source point we only draw part of the LSD. We actually remove a small percentage of the LSD at each of its extremities. On Figure 13, on the right, we can see the artifact caused by the selection of the source point on the border of the bottom knot section (the two endsections are triangulated without any interior point).

Self intersecting objects. Figure 13 shows that our LSD algorithm can handle self-intersecting objects. This would not be the case if we had chosen a height function instead of the shortest path distance. The Medial Axis transform would also fail 


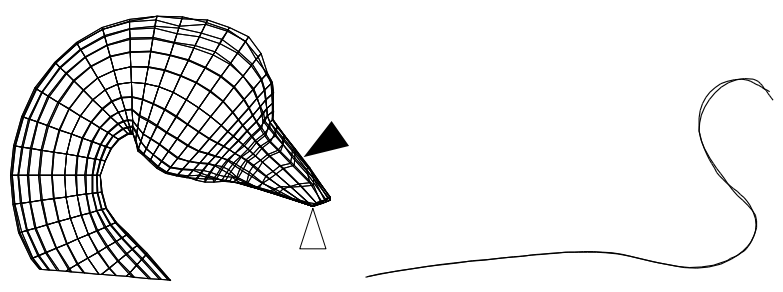

Figure 12: Left: the white and black arrows designate the two source points used to compute the LSDs. Right: the two corresponding LSDs are almost identical.

to extract the correct structure of a self intersecting object. The reason is that shortest path distances only rely on edge connectivity and lengths (the metric) as opposed to a proper embedding of the vertices.
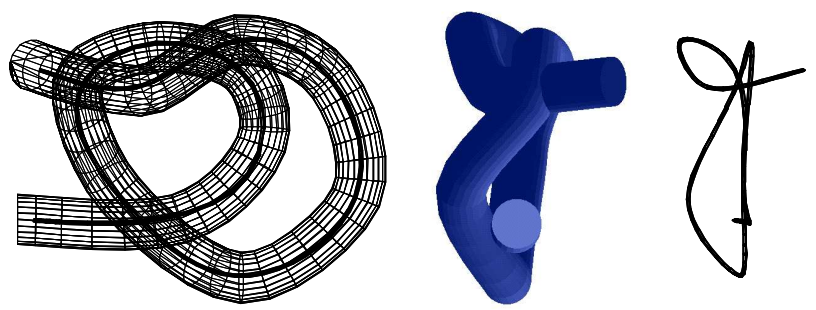

Figure 13: Left: front view of a self intersecting knot and its LSD. Middle: lateral shaded view of the knot. Left: the LSD seen from the same viewpoint.

Extension to non triangulated objects. Notice that the swan and the knot on Figures 11 and 13 are not triangulated. In [12] an extension for extracting LSDs of non triangular meshes is proposed. In a first step we add virtual edges to the polyhedron graph so that the restriction of the new graph to any face is a complete graph. The shortest path distance is computed with this new graph. The definition of a cross edge is also modified to take into account the fact that a face may have more than two cross-edges (see [12] for more details).

The LSDs of the man, the dolphin and the horse ${ }^{3}$ of Figures 14, 15 and 16 have branches. 16 level have been used for the horse, 31 for the dolphin and 50 for the man.

\section{Discussions and Future Work}

We have described an efficient algorithm for constructing the LSD associated to a genus-0 polyhedron whose vertices are valued by the shortest path distance to a source point. This algorithm is fast, robust, and easy to implement. It is mainly designed for tubular and branching objects although it works

\footnotetext{
${ }^{3}$ Copyright 1988, Rhythm \& Hues Studios, Inc.
}
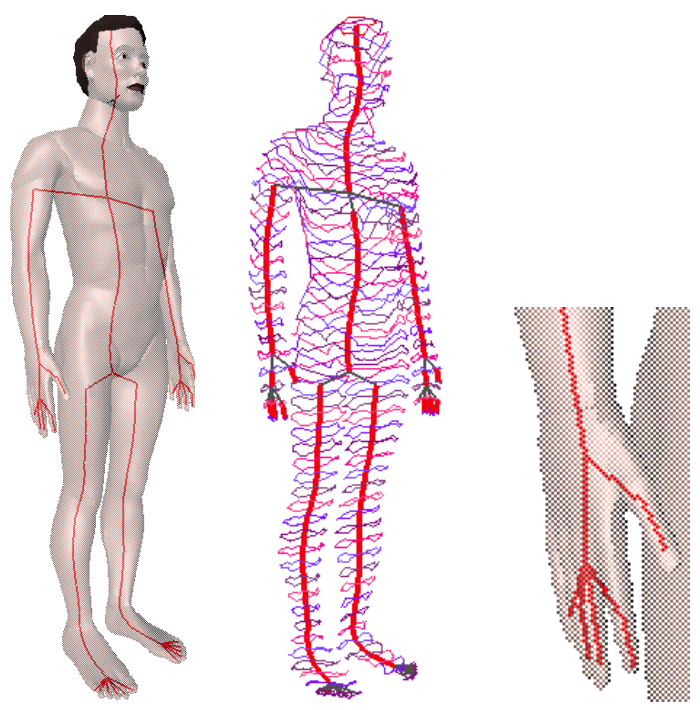

Figure 14: The man (left) is composed of 14946 faces. The source point was selected on the top of the man's head. The LSD (middle) was computed in 7.74 seconds on a SGI Indigo. Details of the LSD is shown on the right.

for any kind of shape. If the object has no obvious tree structure, as for the case of a spherical object, the LSD algorithm still produces a one dimensional structure. Depending on the application this may or may not be useful.

Note that the LSD may lie outside the object. Consider a punched sphere, forming a bowl, with the source point on its bottom (see Figure 17, left). The level sets are circles propagating from the source point up to the rim of the bowl and further shrinking down to the bottom on the interior surface of the bowl. The LSD of this bowl is composed of a straight line going outside the bowl interior up to the rim level, and turning back to the bottom. Although this might not correspond to the intuitive idea of a skeleton this LSD can be used for deforming the bowl

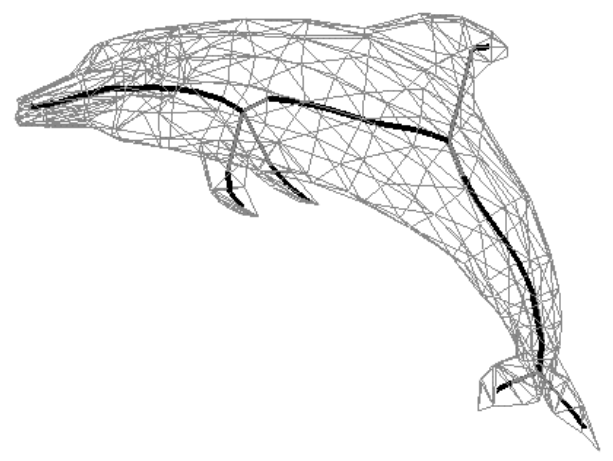

Figure 15: The dolphin is composed of 563 faces and the LSD was computed in 0.25 seconds on a SGI Indigo. 

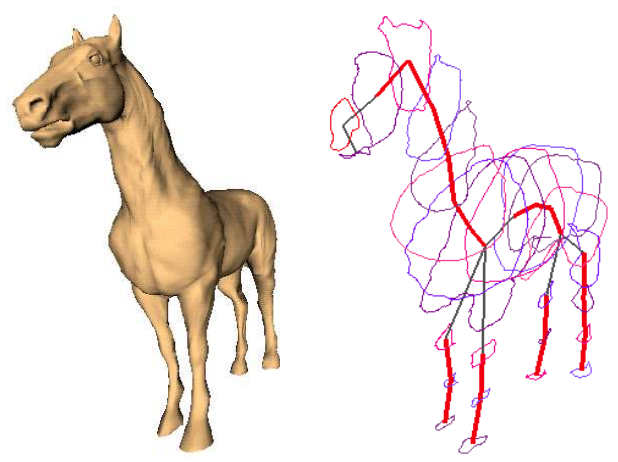

Figure 16: A horse and its LSD. The horse is composed of 22258 faces and the computation of the LSD lasted $5.41 \mathrm{sec}-$ onds on a SGI Indigo.

(see Figure 17) with an axial deformation tool [13]. Generally

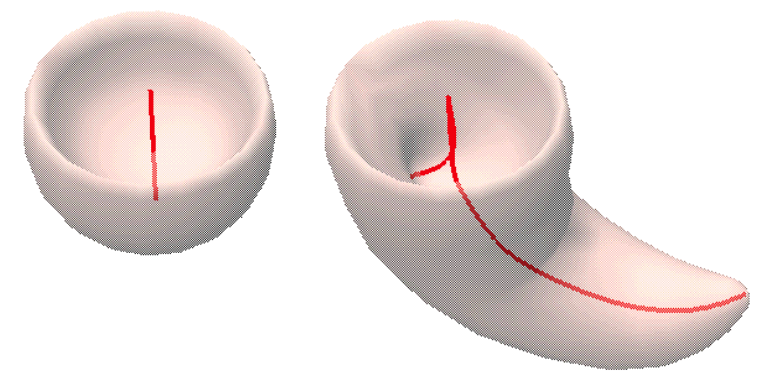

Figure 17: Left: the bowl and its corresponding LSD. Right: the deformed bowl after bending the LSD.

speaking, the LSD provides a set of tubular parameterizations together with articulations that can be used for animation systems. For instance, Walter and Fournier's animation System [24] could certainly take advantage of the LSD.

Finding axial structures is also helpful for extraction of surfaces from scattered point sets (see [18]). In [23] we propose an algorithm, based on the level set paradigm, for extracting skeletal curves from 3D scattered data. It is especially relevant for medical data such as blood vessels.

As presented here, our algorithm in Section 5.1 only applies to 0 -genus surface. Following van Kreveld et al. [22], this could easily be extended to construct LSDs of non- 0 genus surfaces. Some minor modifications are to be made in order to get a starting cross-edge in each contour of a level set. In the case of a 0 genus surface, the level set just below a saddle vertex is composed of a unique contour (contours can only split). It follows that a single decreasing path from a saddle vertex is sufficient to get a starting cross-edge for any contour below a saddle vertex. In Section 5 this decreasing path is provided by the $\pi$ pointer. In the case of a non- 0 genus surface, however, contours can also merge at a saddle vertex. We thus need to obtain starting crossedges in every branch below such a saddle vertex. This can be done by considering as many decreasing paths as there are sequences of "-" in the cycle of neighbors of the saddle vertex (see Figure 7 on the left).

Finally, when applied to scattered point sets, coupling our technique with the feature-based surface generation developed by Takahashi et al. [21] would provide an efficient reconstruction tool.

\section{Acnowledgements}

The authors would like to thank the anonymous reviewers for their valuable comments and for providing useful references $([2,7,22])$.

\section{References}

[1] D. Attali and A. Montanvert. Computing and simplifying 2D and 3D semicontinuous skeletons of $2 \mathrm{D}$ and $3 \mathrm{D}$ shapes. Computer Vision and Image Understanding, 67(3):261-273, September 1997.

[2] U. Axen and H. Edelsbrunner. Auditory morse analysis of triangulated manifolds. In Mathematical Visualization - Algorithms, Applications and Numerics, pages 223-236. Springer-Verlag, 1998.

[3] T. F. Banchoff. Critical points and curvature for embedded polyhedral surfaces. American Mathematical Monthly, 77:475-485, 1970.

[4] B.G. Baumgart. A polyhedron representation for computer vision. In AFIPS Conference, volume 44, pages 589-596, 1975.

[5] H. Blum and R. N. Nagel. Shape description using weighted symmetric axis features. Pattern Recognition, 10:167-180, 1978.

[6] T. H. Cormen, C. E. Leiserson, and R. L. Rivest. Introduction to Algorithms. McGraw-Hill, New York, NY, 1990.

[7] M. de Berg and M. van Kreveld. Trekking the alps without freezing or getting tired. In 1st Annual European Symposium in Algorithms (ESA '93), Lecture notes in Computer Science, volume 726, pages 121-132. Springer Verlag, 1993.

[8] P.H. Eilers. Smoothing and interpolation with finite differences. In Graphics Gems IV. Academic Press, 1994.

[9] J.A. Goldak, X. Yu, A. Knight, and L. Dong. Constructing discrete medial axis of 3D objects. International Journal of Computational Geometry and Applications, 1(3):327-339, 1991.

[10] J.C. Hart. Morse theory for implicit surface modeling. In Visualization and Mathematics '97, Berlin-Dahlem, September 1997.

[11] M. Henle. A Combinatorial Introduction to Topology. Freeman W.H., San Francisco, 1979.

[12] F. Lazarus. Courbes, cylindres et métamorphoses pour l'image de synthèse. Thèse de doctorat en sciences, Université PARIS VII, December 1995. http://www-sic.univ-poitiers.fr/ lazarus/publications.html\#these.

[13] F. Lazarus, S. Coquillart, and P. Jancène. Axial deformations: an intuitive deformation technique. Computer-Aided Design, 26(8):607-613, August 1994.

[14] F. Leymarie and M.D. Levine. Snakes and skeletons. Technical Report TR-CIM-89-3, McGill Research Centre for Intelligent Machines, January 1989.

[15] J. Milnor. Morse theory, volume 51 of Annual of Mathematics Studies. Princeton University Press, Princeton, NJ, 1963.

[16] J.S.B. Mitchell, D.M. Mount, and C.H. Papadimitriou. The discrete geodesic problem. SIAM J. Comput., 16:647-668, 1987.

[17] P.J. Schneider. An algorithm for automatically fitting digitized curves. In Graphics Gems. Academic Press, 1990. 
[18] Y. Shinagawa and T.L. Kunii. Constructing a Reeb graph automatically from cross sections. IEEE Computer Graphics and Applications, 11(6):44 51, November 1991.

[19] Y. Shinagawa, T.L. Kunii, and Y. L. Kergosien. Surface coding based on Morse theory. IEEE Computer Graphics and Applications, 11(5):66-78, September 1991.

[20] S. Takahashi, T. Ikeda, Y. Shinagawa, T.L. Kunii, and M. Ueda. Algorithms for extracting correct critical points and constructing topological graphs from discrete geographical elevation data. In Eurographics'95, pages C181-C-192, Maastrich, The Netherlands, September 1995.

[21] S. Takahashi, Y. Shinagawa, and T.L. Kunii. A feature-based approach for smooth surfaces. In Solid Modeling'97, pages 97-110, Atlanta, Georgia, May 1997. ACM.

[22] M. van Kreveld, R. van Oostrum, C. Bajaj, V. Pascucci, and D. Schikore. Contour trees and small seed sets for isosurface traversal. In Computational Geometry 1997, pages 212-219, 1997.

[23] A. Verroust and F. Lazarus. Extracting skeletal curves from 3D scattered data. In Shape Modeling International'99, Aizu, Japan, March 1999.

[24] M. Walter and A. Fournier. Growing and animating polygonal models of animals. In Eurographics'97, Budapest, HU, September 1997.

[25] K. Weiler. Edge-based data structures for solid modeling in curved-surface environments. IEEE Computer Graphics and Applications, 5(1):21-40, January 1985 . 